Review Article

\title{
Matching and Coordination among Elderly Services, Aging, and Economy in China
}

\author{
Xiaodong Cui $\mathbb{i D}^{1}$ and Ching-Ter Chang $\mathbb{D i D}^{2,3,4}$ \\ ${ }^{1}$ Business College, Nanjing Xiaozhuang University, Nanjing, China \\ ${ }^{2}$ Department of Information Management, Chang Gung University, Taoyuan City, Taiwan \\ ${ }^{3}$ Clinical Trial Center, Chang Gung Memorial Hospital at Linkou, Taoyuan, Taiwan \\ ${ }^{4}$ Department of Industrial Engineering and Management, Ming Chi University of Technology, Taipei, Taiwan \\ Correspondence should be addressed to Ching-Ter Chang; chingter@mail.cgu.edu.tw
}

Received 11 January 2021; Revised 5 March 2021; Accepted 26 March 2021; Published 12 April 2021

Academic Editor: Prashant Kumar Jamwal

Copyright (C) 2021 Xiaodong Cui and Ching-Ter Chang. This is an open access article distributed under the Creative Commons Attribution License, which permits unrestricted use, distribution, and reproduction in any medium, provided the original work is properly cited.

\begin{abstract}
Under the background of China getting old before getting rich, the elderly service, as a public project, should be coordinated with the degree of aging and economic level to avoid the improper allocation of public resources. This article aims to investigate whether an insufficient or overthreshold phenomenon exists in China. Firstly, based on the "active aging theory," the elderly service index is constructed, and then matching and coordination models are proposed to obtain coordination degrees among aging, the elderly service, and the economy. In terms of coordination, elderly service-aging is at a low level and elderly serviceeconomic level shows an agglomeration of high in the east and low in the west, while the elderly service-aging-economic level is quite different among provinces. In provinces with high coordination (e.g., Beijing and Guangdong), the elderly services are appropriate. In comparison, in provinces with low coordination, the elderly services may either lag behind the aging degree (e.g., Sichuan) or exceed the local economic level (e.g., Shannxi). Therefore, the development of aging services should focus on coordination rather than quantification and scale. Considering the regional heterogeneity, we can formulate soft constraints allowing a lower minimum limit and greater flexibility range.
\end{abstract}

\section{Introduction and Literature Review}

Aging is a common challenge faced by all countries in the world. As the largest developing country in the world, how to protect the rights of the elderly has been continuously explored by the Chinese government. For this issue, the $19^{\text {th }}$ Chinese Government Work Report (2017) recently put forward a proposal to accelerate the development of elderly services [1]. At the same time, given the national conditions of aging before getting rich, Mid-Term and Long-Term Plan for Active Response to Population Aging (2019) proposed that the elderly service should not only meet the needs of aging, but also coordinate with the level of economy to maintain the effective and orderly allocation of public financial resources [2].

However, we found that there is a phenomenon of insufficient or overthreshold development of elderly services in
China. For example, in 2018, Shandong and Sichuan have the worst aging, but their development levels of elderly services were ranked the 8th and 11th, which is obviously insufficient. In contrast, elderly services in Shaanxi were ranked the 10th, while its aging degree and economic development level were ranked the 19th and 20th, indicating that the pace of elderly services exceeds the demand for aging, and the local economic level is also not sufficient to support its development. Since elderly services, in the context of overdevelopment or underdevelopment, could lead to disordered and ineffective resources allocation, therefore, we ask two questions: What is the current development of elderly services in China? Do provinces still have mismatch and imbalance problems? In this paper, matching and coupling coordination degree models are used to test the above issues by investigating the matching and coordination among the elderly service, aging, and economic 
levels at the provincial level, which provides empirical references for evaluating the adequacy and sustainability of the elderly service.

With the increase of aging, the research of related elderly service has gradually increased. Earlier studies primarily focused on their theoretical aspects, development status, optimization, and policies [3-6]. Social theory on aging has evolved along with people's attitudes toward their aging society, roughly from "successful aging" to "healthy aging" and further to "active aging". Different from "successful aging" and "healthy aging", the "active aging" not only emphasizes the health status of physical, psychological, and social functions, but also changes the focus of strategic planning from "demand-based" to "rightsbased" to further optimize access to health, participation, and security of the elderly. This is a new development strategy for the international community to deal with aging societies [7].

With promotions of the elderly services in various regions of China, the problem in connection to the regional imbalance in the development of the elderly services and the construction of pension system becomes increasingly salient. Some research has shifted to regional differences of elderly services in China. Fan and Wang [8] established an evaluation system to highlight "economically secure, healthy, productive, intellectual, well educated, and happy aging" quantitatively surveyed the development of elderly services in 31 provinces (cities) in China and identified significant regional imbalances, by applying factor analysis and cluster analysis. Ren [9] established a model to investigate regional differences, evolution features, and balance between intrasystem elements of the development of elderly services. Other research has focused on evaluating and comparing supporting environmental elements for elderly services, such as quality of life of the aged and satisfaction with communities [10], health and environment of the elderly [11], and a pension model for integration and innovation [12].

Elderly services refer to the government's commitments to providing services to the elderly, and economic strength is the foundation for the development of elderly services [13]. Therefore, the development of elderly services should be appropriate for both the level of aging and the local economic development level. Only appropriate coordinated development among aging, elderly services, and economy level can maintain quality of life for the elderly along with efficient resource allocations. However, there are few quantitative studies on the coordinated development of these issues at the same time. Zeng et al. [14] did one of the few studies on the matching between elderly services and economic development. Their article uses the center of gravity and spatial overlap model to calculate the geographical coordinates and mismatch distance between the elderly services and the economy, finding the regional characteristics of the two misalignments. The literature focuses more on the geographical perspective, with less research on the interaction and coordination between elderly services and economic conditions. Studies on the development of elderly services without considering needs of the elderly may be limited to quantitative objectives, leading to ineffective resource allocation. This paper thus explores coordination among elderly services, aging, and economic level at same time in provinces (cities) of China.

Research on coordination relations mainly relies on the concept of coordination in physics, which has been widely applied to analyze the intersystem coordinated development [15-17]. Based on this theory, this paper attempts to analyze the coordination relation among elderly services, aging, and economic levels in provinces (cities) of China. Firstly, an evaluation index system of elderly services is constructed and indices of the system are calculated, and then matching statistics and coordination models are used to analyze coordination relation and the time-varying characteristics and spatial differences. Compared with the existing literature, the contributions of this paper are as follows: first, based on the "active aging theory" of the international aging society, from the three dimensions of health, participation, and security, we construct an index system of elderly service development, which has stronger international comparability; second, from the coordination development perspective, the matching and coordination methods are used to evaluate the development level of the elderly services, which provides a new and more appropriate evaluation standard; third, examining the development trend and regional differences of the elderly services from the time and space dimensions provides a reference for the planning of elderly services to match with local aging and economic level.

\section{Matching Degree among Elderly Services, Aging, and Economic Levels}

This article takes the administrative units of 22 provinces (cities) in China as the research object, and the research data was collected from the "China Statistical Yearbook", "China Civil Affairs Statistical Yearbook", "National Economic Development and Social Statistics Announcement", and statistical yearbooks on national economic development of provinces (cities) for years 2009, 2012, 2015, and 2018.

For the convenience of presentation, symbols and notations used in this paper are shown in Table 1.

2.1. Composite Index of the Elderly Services. Currently, there are no agreed-upon evaluation criteria for the indices of the development of elderly services. From the perspective of "active aging theory" in the international aging society and with reference to existing literature [8,9], this paper uses eight indices in three dimensions (i.e., health, security, and participation) for the development of elderly services as shown in columns 1 and 2 in Table 2.

Due to the different indicator units, referring to the research method of Li [18], the range method is used to nondimensionalize the original data, and then linear weighting is used to calculate the comprehensive index, in which the entropy method is used to determine the indicator weight to avoid subjective assignment problem [19]. The specific calculation process is shown in Figure 1 and index weights are shown in column 3 of Table 2. 
TABle 1: Notations definition.

\begin{tabular}{|c|c|}
\hline Symbols & Notations \\
\hline$i$ & $i$ th province (city), $i=1,2, \ldots, m$ \\
\hline$j$ & $j$ th elderly services, $j=1,2, \ldots, n$ \\
\hline$x_{i j}$ & The value of $j$ th elderly services in $i$ th province (city) \\
\hline$w_{j}$ & The weight of $j$ th elderly services \\
\hline \multicolumn{2}{|c|}{ The index notation } \\
\hline $\begin{array}{l}U_{1 i} \\
U_{2 i} \\
U_{3 i}\end{array}$ & $\begin{array}{l}U_{1 i} \text { is the elderly services system with the index of } U_{1} \text { for } i \text { th province (city) } \\
U_{2 i} \text { is the aging system with the index of } U_{2} \text { for } i \text { th province (city) } \\
U_{3 i} \text { is the economic system with the index of } U_{3} \text { for } i \text { th province (city) }\end{array}$ \\
\hline \multicolumn{2}{|c|}{ Matching and coordination } \\
\hline 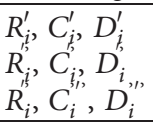 & $\begin{array}{l}\text { Matching degree, coupling degree, and coordination degree, respectively, in elderly services-aging for ith province (city) } \\
\text { Matching degree, coupling degree, and coordination degree, respectively, in economic-elderly services for } i \text { th province (city) } \\
\text { Matching degree, coupling degree, and coordination degree in elderly services-aging-economic level for } i \text { th province (city) }\end{array}$ \\
\hline
\end{tabular}

TABLE 2: Indices of the elderly services and weights.

\begin{tabular}{|c|c|c|c|c|c|}
\hline \multirow{2}{*}{ Dimension (1) } & \multirow{2}{*}{ Index meaning and unit (2) } & \multicolumn{4}{|c|}{ Weight in different years (3) } \\
\hline & & 2009 & 2012 & 2015 & 2018 \\
\hline \multirow{3}{*}{ Health } & Number of health workers per 1,000 aged people & 0.100 & 0.117 & 0.144 & 0.137 \\
\hline & Number of hospitals per 100,000 aged people & 0.140 & 0.181 & 0.181 & 0.166 \\
\hline & Urban basic medical insurance per capita & 0.183 & 0.143 & 0.142 & 0.112 \\
\hline \multirow{3}{*}{ Security } & Number of old age service beds per 1,000 aged people & 0.132 & 0.138 & 0.186 & 0.188 \\
\hline & Urban employees basic endowment insurance $(10,000 \mathrm{RMB}$ per person) & 0.080 & 0.072 & 0.078 & 0.085 \\
\hline & Participation in the urban and rural old age insurance (\%) & 0.326 & 0.310 & 0.231 & 0.269 \\
\hline \multirow{2}{*}{ Participation } & Senior citizen school enrollment $(\%)$ & 0.016 & 0.018 & 0.020 & 0.023 \\
\hline & Coverage of senior citizen activity center per 1,000 people & 0.023 & 0.022 & 0.019 & 0.020 \\
\hline
\end{tabular}

2.2. Measure of Matching Degree. An appropriate study on coordinated development should focus on both matching and coordination levels. The matching degree can intuitively reflect the deviation of intersystem development level by the double-index and single-index methods. This article examines the matching degree in elderly services $\left(U_{1 i}\right)$, aging $\left(U_{2 i}\right)$, and economic level $\left(U_{3 i}\right)$ as follows, in which $U_{2 i}$ denotes the proportion of aged 65 and above in the total population and $U_{3 i}$ denotes the GDP per capita after price index adjustment.

2.2.1. Double-Index Test. The double-index method combines $U_{1 i}$ and $U_{2 i}$ (or $U_{3 i}$ ) to measure the matching degree. Based on the index value, $U_{1 i}, U_{2 i}$, and $U_{3 i}$ are divided into the two levels of high (value of the index $>$ mean) and low (value of the index < mean), yielding four sections: dualhigh, dual-low, single high (low), and single low (high) as shown in Table 3.

As seen in the upper part of Table 3, referring to mismatching types in elderly services-aging, SH, JS, LN, and SC are consistently with dual-high, and GD, FJ, JX, and HN are consistently with dual-low, indicating that the development level of elderly services in these provinces (cities) can be appropriate to the degree of aging, while the development level of elderly services in other regions may be insufficient or overly advanced. Although the number of mismatched provinces (cities) has decreased in recent years, it still exceeds $1 / 4$ of the total, indicating that mismatches are still relatively common. The matching type does not show a certain regional regularity. The double-high type includes both developed regions (e.g., SH and ZJ) as well as underdeveloped provinces (e.g., LN and SC). This also indirectly indicates that the development of elderly services not only relates to economic development, but also relates to local government policies, administrative orientation, structure, and other regional characteristics.

The lower part of Table 3 lists the mismatch in economicelderly services by the double-index method. In the time dimension, SH, BJ, TJ, and ZJ are consistently with dualhigh, while HUN, GX, JX, and HN are consistently with dual-low. Other provinces (cities) also show roughly stable mismatch type. The proportion of dual-high and dual-low types of provinces (cities) is high, and there are few mismatched provinces (cities) with regional features. Most dualhigh provinces (cities) are in more developed eastern areas, and most dual-low provinces (cities) are in the central and western regions. These features are consistent with observation and the conclusions of prior study [14].

2.2.2. Single-Index Test. The double-index method intuitively shows the matching degree and distinguishes regions with significant mismatch to some extent. However, the dual-index method cannot quantify the degree of mismatch, so a single-index method was used for this analysis, with $\mathbf{R}$ for direct measure. After the dimensionless treatment and normalization of the indices, we obtain the single mismatch index $R_{i}^{\prime}$ in elderly services-aging, $R_{i}^{\prime \prime}$ in economic-elderly services, and $R_{i}^{\prime \prime}$ in elderly services-aging-economic level as follows: 


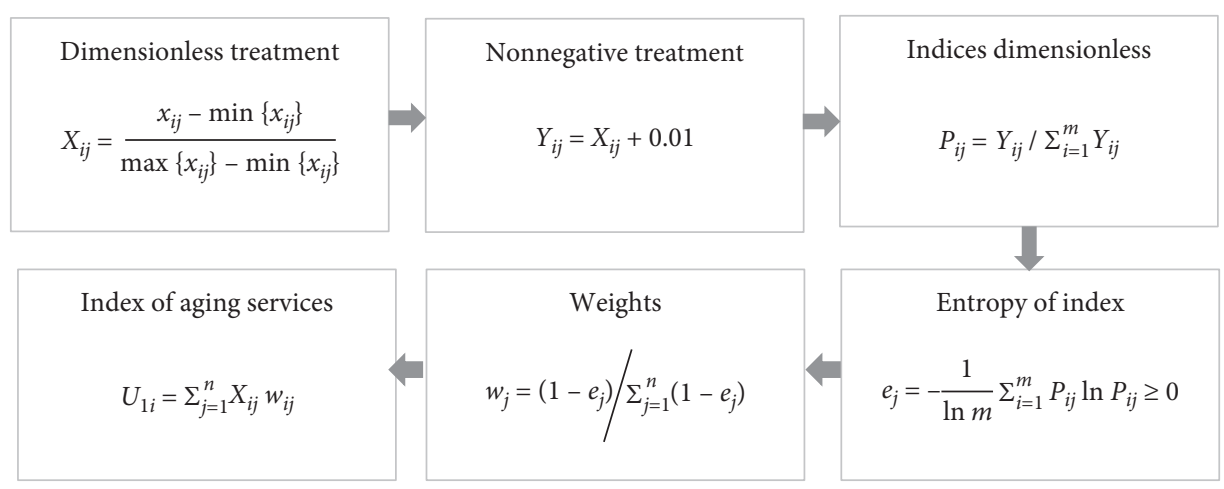

FIgURE 1: The calculation steps of elderly services index.

TABLE 3: Matching type of 22 provinces (cities) in different years.

\begin{tabular}{|c|c|c|c|c|}
\hline \multicolumn{5}{|c|}{ Matching type in elderly services-aging } \\
\hline Type & 2009 & 2012 & 2015 & 2018 \\
\hline Double high & SH, JS, LN,TJ, ZJ, JL, CQ & SH, JS, LN, SC,TJ, SD & SH, JS, LN, SC, ZJ, HB & $\begin{array}{l}\text { SH, JS, LN, SC, ZJ, HB, } \\
\text { SD, HB }\end{array}$ \\
\hline Double low & GD, JX, HN, GX, SD HUB, HB & GD, JX, HN, FJ, HB, JL & GD, JX, HN, FJ, GX & $\begin{array}{c}\text { GD, JX, HN, FJ, SX, GX, } \\
\text { HLJ, JL }\end{array}$ \\
\hline $\begin{array}{l}U_{1} \text { high, } U_{2} \\
\text { low }\end{array}$ & BJ, SAX, SX, HLJ & BJ, SAX, SX, ZJ, HLJ & BJ, SAX, SX, TJ, HLJ & BJ, SAX, TJ \\
\hline $\begin{array}{l}U_{1} \text { low, } U_{2} \\
\text { high }\end{array}$ & $\mathrm{HN}, \mathrm{AH}, \mathrm{SC}, \mathrm{FJ}$ & $\mathrm{HN}, \mathrm{AH}, \mathrm{HB}, \mathrm{CQ}, \mathrm{GX}$ & $\mathrm{HN}, \mathrm{AH}, \mathrm{JL}, \mathrm{HB}, \mathrm{SD}, \mathrm{CQ}$ & $\mathrm{HN}, \mathrm{AH}, \mathrm{CQ}$ \\
\hline \multicolumn{5}{|c|}{ Matching type in economic-elderly services } \\
\hline Type & 2009 & 2012 & 2015 & 2018 \\
\hline Double high & SH, TJ, BJ, ZJ, LN, JL, JS & SH, BJ, TJ, LN, ZJ, JS, SD & SH, BJ, TJ, ZJ, LN, JS & $\begin{array}{c}\text { SH, BJ, TJ, ZJ, JS, SD, HB, } \\
\text { SAX }\end{array}$ \\
\hline Double low & $\begin{array}{c}\text { HUN, GX, JX, HN, HB, AH, } \\
\text { SC, HUB }\end{array}$ & $\begin{array}{c}\text { HUN, GX, JX, HN, HUB, HB, } \\
\text { CQ, AH }\end{array}$ & $\begin{array}{c}\text { HUN, GX, JX, HN, HUB, } \\
\text { HB, AH, JL }\end{array}$ & $\begin{array}{c}\text { HUN, GX, JX, HN,JL, } \\
\text { AH, HLJ }\end{array}$ \\
\hline $\begin{array}{l}U_{1} \text { high, } \\
U_{2} \text { low }\end{array}$ & SX,,SAX, HLJ, CQ & SX, SAX, HLJ, SC & SX, SAX, HLJ, SC & SX, HUB, LN, SC \\
\hline $\begin{array}{l}U_{1} \text { low } \\
U_{2} \text { high }\end{array}$ & HSD, FJ, GD & JL, FJ, GD & $\mathrm{GD}, \mathrm{FJ}, \mathrm{CQ}, \mathrm{SD}$ & $\mathrm{CQ}, \mathrm{GD}, \mathrm{FJ}$ \\
\hline
\end{tabular}

$$
\begin{aligned}
& R_{i}^{\prime}=U_{1 i}-U_{2 i}, \\
& R_{i}^{\prime \prime}=U_{3 i}-U_{1 i}, \\
& R_{i}^{\prime \prime}=R_{i}^{\prime}-R_{i}^{\prime \prime} .
\end{aligned}
$$

To further reveal the degree of mismatch, this study uses the method of Bao and Chen [20] to divide mismatching states into six sections according to a mean single index: positive high $(R \geq 1)$, positive medium $(0.3 \leq R<1)$, positive low $(0 \leq R<0.3)$, negative high $(R \leq-1)$, negative medium $(-1 \leq R<0.3)$, and negative low $(-0.3 \leq R<0)$. The means for single-index $R_{i}^{\prime}, R_{i}^{\prime \prime}$, and $R_{i}^{\prime \prime}$ are shown in Figure 2, and the matching types and regional characteristics are shown in Table 4 and Figure 3.

And the following conclusions can be obtained:

(1) About matching degree $R_{i}^{\prime}$ in elderly services-aging: as shown in Figure 2 and column 2 in Table 4, the mismatch between elderly services and aging is widespread in 22 provinces (cities). In particular, about 16 provinces (cities) have a high and medium mismatch index, and only seven provinces have a relatively low mismatch index. In terms of mismatch direction, BJ, SH, GD, and SX are in the positive high mismatch section with a mismatch index greater than 1, representing a large deviation between elderly services and aging degree, i.e., faster development of elderly services than degree of population aging in these regions. AH, SC, and $\mathrm{CQ}$ are in the negative high mismatch section with a mismatch index less than -1 , i.e., indicating less development of elderly services than the degree of population aging. In terms of regional features of match types as shown in Figure 3, there is no apparent pattern. But it should be noted that JS and SD, with relatively high economic development, are negative medium, while SX is positive high section although it is less economically developed. Therefore, economic conditions and 

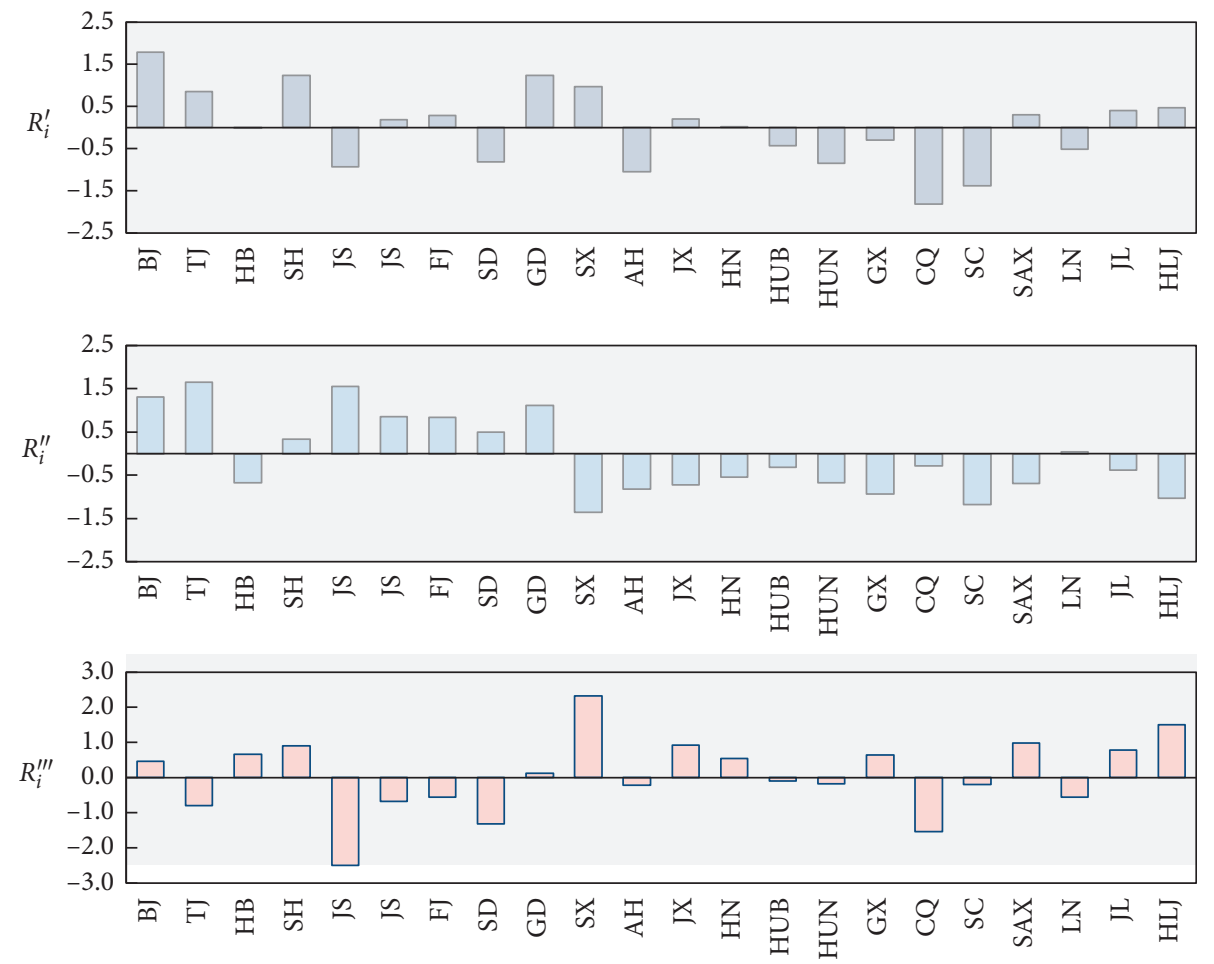

FIgURE 2: Single-index of 22 provinces (cities).

Table 4: Matching types of 22 provinces (cities).

\begin{tabular}{lccc}
\hline Type (1 ) & Elderly services-aging ( 2 $)$ & Economic level-elderly services ( 3 ) & Elderly services-aging-economic level ( 4 ) \\
\hline$R \geq 1$ & BJ, SH, GD, SX & BJ, TJ, JS, GD & SX, SAX, HLJ \\
$0.3 \leq R<1$ & TJ, JL, HLJ & SH, ZJ, FJ, SD & BJ, HB, SH, JX, HN, GX, JL \\
$0 \leq R<0.3$ & ZJ, FJ, JX, HN, SAX & LN & GD \\
$-0.3 \leq R<0$ & HB, GX & CQ & AH, HUB, HUN, SC \\
$-1 \leq R<-0.3$ & JS, SD, HUB, HUN, LN & HB, AH, JX, HN, HUB, HUN, GX, SAX, JL & TJ, ZJ, FJ, LN \\
$R \leq-1$ & AH, CQ, SC & SX, SC, HLJ & JS, SD, CQ \\
\hline
\end{tabular}

elderly services should be considered together to determine whether elderly services are over- or underdeveloped.

(2) About matching degree in economic-elderly services: as shown in Figure 2 and column 3 in Table 4, nine provinces (cities) are within positive sections and 13 provinces (cities) are within negative sections. Four provinces (cities), including BJ, TJ, JS, and GD are within the positive high section with an index greater than 1, indicating significant mismatch between the development of elderly services and local economic level. LN and CQ show a good matching degree. SX, SC, and HLJ are negative high, with a mismatch index less than -1 , indicating the local economic conditions exceed the development of elderly services. Among the nine provinces (cities) in the negative high mismatch section and the negative medium mismatch section, approximately $60 \%$ of them have overdeveloped elderly services. In terms of regional features of these types as shown in Figure 3, the matching index is highly consistent with local economic development. Most provinces (cities) in the positive sections are in the developed eastern areas, while most provinces (cities) in negative sections are in the less developed in the centralwestern provinces (cities).

(3) About matching degree in elderly services-agingeconomic level: $R_{1}$ reflects the adequacy of elderly services to the degree of population aging, $R_{2}$ reflects the efficiency of the elderly services to economic development level, and only the combination of the two of them can reflect the appropriateness of the elderly services. For example, GD falls within the positive high section in both of them, which indicates that despite the fact that the development of elderly services is higher than that of aging, the economic development is also higher than that of elderly 


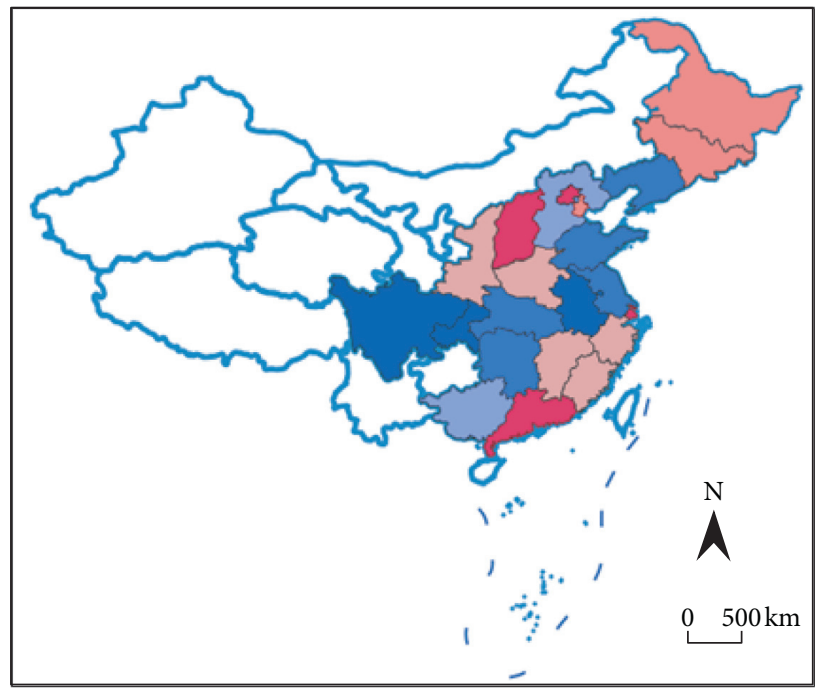

Elderly services-aging

$R \geq 1$

$0.3 \leq R<1$

$0 \leq R<0.3$

$-0.3 \leq R<0$

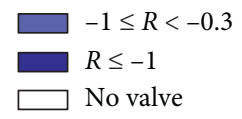

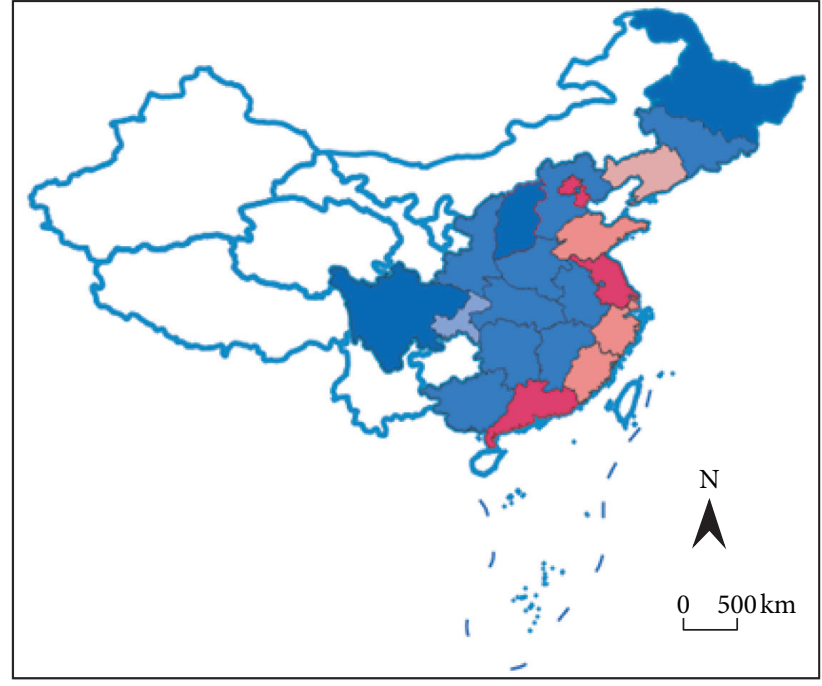

Economic-elderly services

$\square \geq 1$
$\square 0.3 \leq R<1$
$\square 0 \leq R<0.3$

$\square-1 \leq R<-0.3$

$R \leq-1$

$\square$ No valve

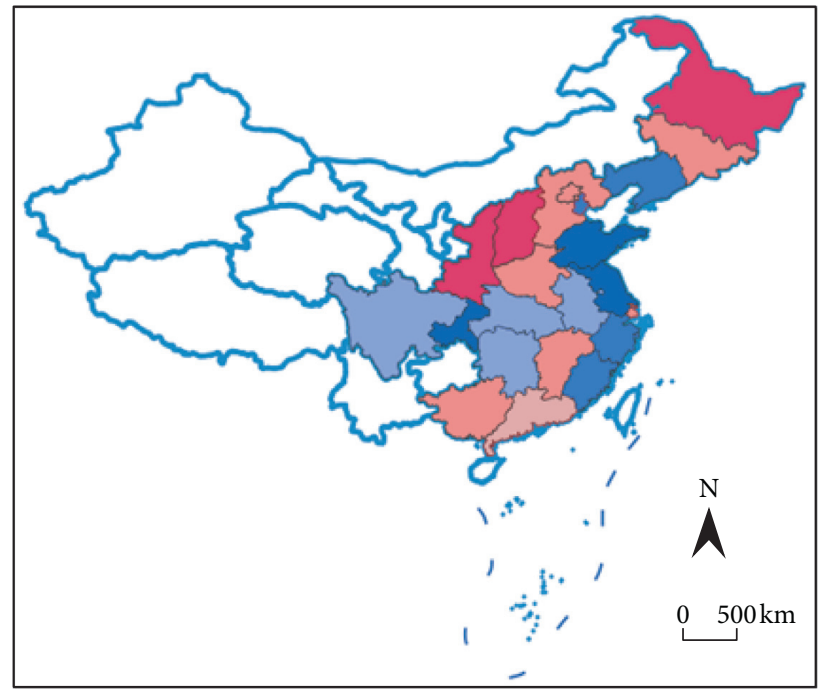

Elderly services-aging-economic

$\square \geq 1$
$\square .3 \leq R<1$
$\square \leq R<0.3$
$\square-0.3 \leq R<0$

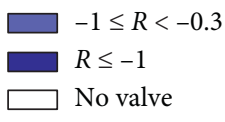

FIGURE 3: Regional characteristics of matching degree.

services; that is, its economic power is sufficient to support GD to develop elderly services appropriately. The same case can be found in AH, HUB, HUN, and SC. SX, SAX, and HLJ fall within the positive high section indicating that the degree of elderly services is overdeveloped, and local economic development in three provinces (SX, SAX, and HLJ) is insufficient to support excess development of elderly services, which may result in inefficient resource utilization. In contrast, in JS, SD, and CQ, elderly services are less 
developed than aging and do not match the local economic level, which together result in underdevelopment of elderly services.

\section{Coordination among Elderly Services, Aging, and Economic Level}

Matching degree analysis can reflect the degree of misalignment in elderly services-aging-economic level, but it is difficult to reflect the level of coordination development, which means that the same matching degree may represent different development levels. For example, BJ, SH, JX, and GX are all within the positive high mismatch section, but the development of the degree of elderly services in $\mathrm{BJ}$ and $\mathrm{SH}$ is far greater than JX and GX as shown in column 4 of Table 4 , so it is necessary to construct an appropriate test model to reflect the degree of matching and development level at the same time.

3.1. Measure of Coordination Degree. Coordination degree as a physics concept, due to reflecting both development and coordination of intersystem, has been widely used in social science research to investigate relationships between two or more systems that are associated with each other but without a cause-and-effect structure [20,21]. Coordination is derived by adding utility to coupling, and coupling degree can be calculated as

$$
\begin{aligned}
& C_{i}^{\prime}=2 \cdot \frac{\sqrt{U_{1 i} \times U_{2 i}}}{\left(U_{1 i}+U_{2 i}\right)}, \\
& C_{i}^{\prime}=2 \cdot \frac{\sqrt{U_{1 i} \times U_{3 i}}}{\left(U_{1 i}+U_{3 i}\right)}, \\
& C_{i}^{\prime}=2 \cdot \frac{\sqrt{R_{i}^{\prime} \times R_{i}^{\prime}}}{\left(R_{i}^{\prime}+R_{i}^{\prime}\right)},
\end{aligned}
$$

where a larger value of $\mathbf{C} \in[0,1]$ indicates greater coupling between two systems.

The coordination degree can reflect the degree of coordination and development at the same time, as shown in

$$
\begin{aligned}
T_{i}^{\prime} & =\alpha U_{1 i}+\beta U_{2 i}, \\
D_{i}^{\prime} & =\sqrt{C_{i}^{\prime} \cdot T_{i}^{\prime}}, \\
T_{i}^{\prime} & =\alpha U_{1 i}+\beta U_{3 i}, \\
D_{i}^{\prime} & =\sqrt{C_{i}^{\prime} \cdot T_{i}^{\prime}}, \\
T_{i}^{\prime} & =\alpha R_{i}^{\prime}+\beta R_{i}^{\prime}, \\
D_{i}^{\prime} & =\sqrt{C_{i}^{\prime} \cdot T_{i}^{\prime}}, \quad D \in[0,1],
\end{aligned}
$$

where a greater value of $\mathbf{D} \in[0,1]$ is used to indicate a higher coordination degree. For simplicity, the two subsystems have equal weight $[22,23]$.
To further reveal the regional characteristics of matching, this paper divides coordination degree into four types: low coordination, basic coordination, good coordination, and high coordination according to Yang et al. [24].

\subsection{Coordination Degree Calculation Results.}

Coordination degrees and coordination types are listed in Tables 5 and 6, and regional characteristics are as shown in Figure 4 .

Based on Tables 5 and 6 and Figure 4, the following conclusions can be obtained:

(1) About coordination degree in elderly services-aging: the coordination degree in elderly services-aging in most provinces (cities) slightly fluctuated from 2009 to 2018 , but coordination type remained relatively stable. The coordination degree in provinces JS, ZJ, and SD has greatly improved. In particular, coordination degree in JS has improved from 0.5447 in 2009 to 0.6121 in 2018, and its coordination type also improved from basic coordination to good coordination. In contrast to coordination in TJ, it has greatly decreased, from 0.7866 in 2009 to 0.5381 in 2018 , and coordination type deteriorated from good to basic. TJ has failed to promote the development of elderly services due to its slow aging (aging level in TJ in 2018 was only slightly higher than that in GD and FJ). The proportions of coordination type in 2009 and 2018 remained roughly stable. More than $60 \%$ of the provinces (cities) have remained at the basic coordination level $(0.4 \leq D<0.6)$, and distribution was approximate. $\mathrm{BJ}$ and $\mathrm{SH}$ have remained in the range of $0.6 \leq D<0.8$, while other provinces including GD, JX, and GX have remained between $0.2 \leq D<0.4$. Overall (as shown in Figure 4), regions with lower population aging have lower coordination, but higher coordination degree depends on both the degree of aging and the level of regional economic development.

(2) About coordination degree in economic level-elderly services: the coordination in most provinces (cities) improved slightly, especially in JS, which improved from basic to good coordination. Overall, nearly half the provinces (cities) have remained at the basic coordination level $(0.2 \leq D<0.4)$, and only a few developed provinces (cities) have remained at good coordination level $(\geq 0.6)$. In addition, most provinces (cities) with low coordination level were in less developed regions of the midwest, while most provinces (cities) with high coordination level were located in Eastern provinces (cities) with high economic development level (as shown in Figure 4). This indicates that economic development is a crucial to the elderly services. Overall coordination level between elderly services and aging was greater than that between economic and elderly services, indicating that, compared with economic conditions, the 
TABle 5: Coordination degree of 22 provinces (cities).

\begin{tabular}{|c|c|c|c|c|c|c|c|c|c|c|c|c|}
\hline \multirow[t]{2}{*}{ Provinces (cities) } & \multicolumn{4}{|c|}{ Elderly services-aging } & \multicolumn{4}{|c|}{ Economic level-elderly services } & \multicolumn{4}{|c|}{$\begin{array}{c}\text { Elderly services-aging-economic } \\
\text { level }\end{array}$} \\
\hline & 2009 & 2012 & 2015 & 2018 & 2009 & 2012 & 2015 & 2018 & 2009 & 2012 & 2015 & 2018 \\
\hline BJ & 0.697 & 0.597 & 0.713 & 0.645 & 0.883 & 0.891 & 0.889 & 0.880 & 0.976 & 0.985 & 0.986 & 0.978 \\
\hline $\mathrm{TJ}$ & 0.787 & 0.703 & 0.652 & 0.538 & 0.834 & 0.871 & 0.856 & 0.744 & 0.899 & 0.993 & 0.997 & 0.946 \\
\hline $\mathrm{HB}$ & 0.390 & 0.453 & 0.476 & 0.542 & 0.389 & 0.374 & 0.314 & 0.322 & 0.802 & 0.712 & 0.633 & 0.592 \\
\hline $\mathrm{SH}$ & 0.910 & 0.656 & 0.870 & 0.879 & 0.956 & 0.905 & 0.931 & 0.938 & 0.905 & 0.948 & 0.863 & 0.804 \\
\hline JS & 0.545 & 0.572 & 0.590 & 0.612 & 0.550 & 0.624 & 0.645 & 0.679 & 0.767 & 0.818 & 0.751 & 0.757 \\
\hline ZJ & 0.578 & 0.485 & 0.586 & 0.649 & 0.615 & 0.628 & 0.640 & 0.686 & 0.869 & 0.922 & 0.835 & 0.775 \\
\hline FJ & 0.434 & 0.419 & 0.410 & 0.367 & 0.430 & 0.499 & 0.506 & 0.526 & 0.797 & 0.865 & 0.880 & 0.883 \\
\hline SD & 0.453 & 0.544 & 0.497 & 0.592 & 0.486 & 0.532 & 0.490 & 0.513 & 0.860 & 0.765 & 0.708 & 0.465 \\
\hline GD & 0.225 & 0.212 & 0.205 & 0.236 & 0.475 & 0.434 & 0.475 & 0.523 & 0.922 & 0.945 & 0.953 & 0.898 \\
\hline SX & 0.402 & 0.493 & 0.468 & 0.435 & 0.437 & 0.399 & 0.187 & 0.279 & 0.594 & 0.563 & 0.558 & 0.535 \\
\hline $\mathrm{AH}$ & 0.416 & 0.430 & 0.417 & 0.507 & 0.204 & 0.235 & 0.240 & 0.300 & 0.520 & 0.598 & 0.563 & 0.497 \\
\hline JX & 0.338 & 0.342 & 0.350 & 0.380 & 0.213 & 0.207 & 0.213 & 0.283 & 0.707 & 0.685 & 0.665 & 0.631 \\
\hline $\mathrm{HN}$ & 0.252 & 0.389 & 0.396 & 0.430 & 0.273 & 0.265 & 0.259 & 0.313 & 0.812 & 0.683 & 0.645 & 0.606 \\
\hline HUB & 0.406 & 0.510 & 0.519 & 0.518 & 0.313 & 0.404 & 0.437 & 0.467 & 0.708 & 0.673 & 0.677 & 0.664 \\
\hline HUN & 0.469 & 0.535 & 0.487 & 0.483 & 0.301 & 0.336 & 0.337 & 0.349 & 0.624 & 0.574 & 0.600 & 0.568 \\
\hline GX & 0.384 & 0.419 & 0.414 & 0.398 & 0.200 & 0.206 & 0.216 & 0.218 & 0.628 & 0.609 & 0.599 & 0.540 \\
\hline CQ & 0.575 & 0.541 & 0.533 & 0.550 & 0.367 & 0.390 & 0.425 & 0.450 & 0.410 & 0.466 & 0.459 & 0.530 \\
\hline SC & 0.550 & 0.599 & 0.602 & 0.578 & 0.240 & 0.271 & 0.311 & 0.331 & 0.457 & 0.468 & 0.441 & 0.399 \\
\hline SAX & 0.496 & 0.526 & 0.523 & 0.467 & 0.365 & 0.445 & 0.431 & 0.447 & 0.661 & 0.685 & 0.686 & 0.699 \\
\hline $\mathrm{LN}$ & 0.580 & 0.563 & 0.600 & 0.558 & 0.531 & 0.611 & 0.564 & 0.415 & 0.748 & 0.835 & 0.694 & 0.536 \\
\hline JL & 0.428 & 0.352 & 0.494 & 0.469 & 0.422 & 0.468 & 0.439 & 0.379 & 0.783 & 0.821 & 0.718 & 0.629 \\
\hline HLJ & 0.427 & 0.486 & 0.547 & 0.470 & 0.393 & 0.405 & 0.337 & 0.234 & 0.754 & 0.666 & 0.567 & 0.513 \\
\hline
\end{tabular}

TABle 6: Coordination types of 22 provinces (cities).

\begin{tabular}{lccc}
\hline System & Classification criteria & Period & Provinces (cities) \\
\hline & Low coordination & 2009 & GD, JX, GX, HN, HB \\
& $0.2 \leq D<0.4$ & 2018 & GD, JX, GX, FJ \\
Elderly services-aging & Basic coordination & 2009 & SX, JL, HLJ, AH, HN, SAX, LN, CQ, SC, SD, JS, ZJ, FJ \\
& $0.4 \leq D<0.6$ & 2018 & SX, JL, HLJ, AH, HN, SAX, LN, CQ, SC, SD, HB, HUN, TJ, HUB \\
& Good coordination & 2009 & BJ, SH, TJ \\
& $0.6 \leq D<0.8$ & 2018 & BJ, SH, JS, ZJ, \\
& Low coordination & 2009 & AH, GX, JX, SC, HN, HUN, HLJ, HB, HUB, CQ, SAX, \\
& $0.2 \leq D<0.4$ & 2018 & AH, GX, JX, SC, HN, HUN, HLJ, HB, SX, JL \\
Elderly services-economic level & Basic coordination & 2009 & FJ, JL, GD, SX, SD, LN, JS \\
& $0.4 \leq D<0.6$ & 2018 & LN, CQ, SAX, HUB, SD, FJ, GD \\
& Good coordination & 2009 & ZJ, TJ \\
& $0.6 \leq D<0.8$ & 2018 & JS, ZJ, TJ \\
& High coordination & 2009 & BJ, SH \\
Elderly services-aging-economic level SH & Good coordination & 2009 & CQ, SC, AH, SX \\
& $0.6 \leq D<0<1$ & 2018 & Bub, SAX, SX, HUN, GX, JX, LN, HLJ, JS, JL, FJ \\
& Bigh coordination & 2009 & HUB, SAX, JL, JX, JS, ZJ, HN \\
& $0.8 \leq D<1$ & 2018 & HB, SD, ZJ, TJ, SH, GD, BJ
\end{tabular}

intensified degree of aging boosted the development of elderly services to a greater extent.

(3) About coordination degree in elderly services-agingeconomic level: overall coordination remained at the basic level $(\geq 0.4)$, indicating that elderly services in almost provinces (cities) have formed appropriate mode of to local conditions. In recent years, however, overall coordination decreased slightly. The number of provinces (cities) at the level of good and above level has decreased from eighteen in 2009 to twelve in
2018, which was partially attributable to faster degree of aging than elderly services. In terms of regional differences (as shown in Figure 4), coordination decreases from the east to the west. Eastern provinces (cities) SH, GD, TJ, and BJ have consistently maintained high coordination, while western provinces CQ, SC, AH, and SX have been at a low level. A high coordination degree indicated that the development of elderly services was appropriate to the aging needs and local economic level. A low 


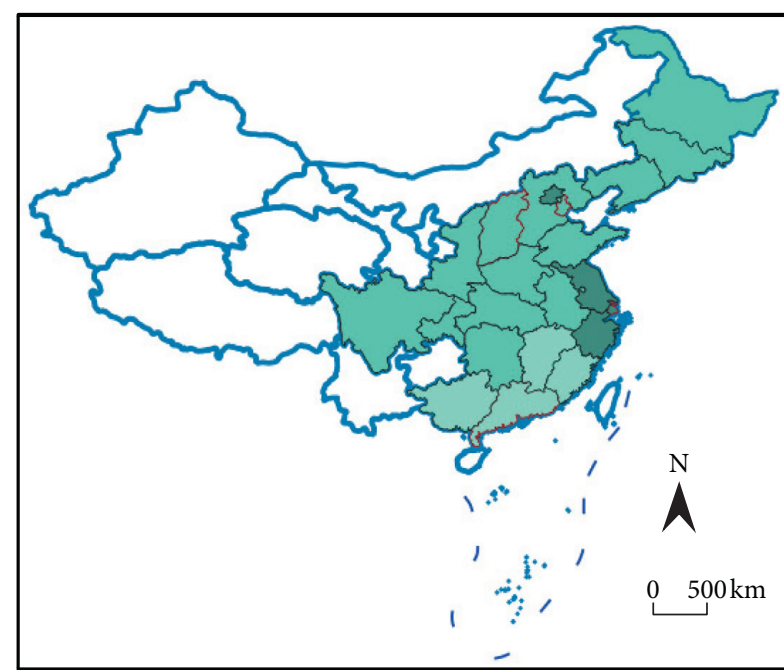

Elderly services-aging

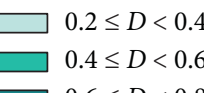

$0.6 \leq D<0.8$

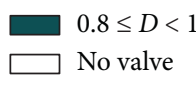

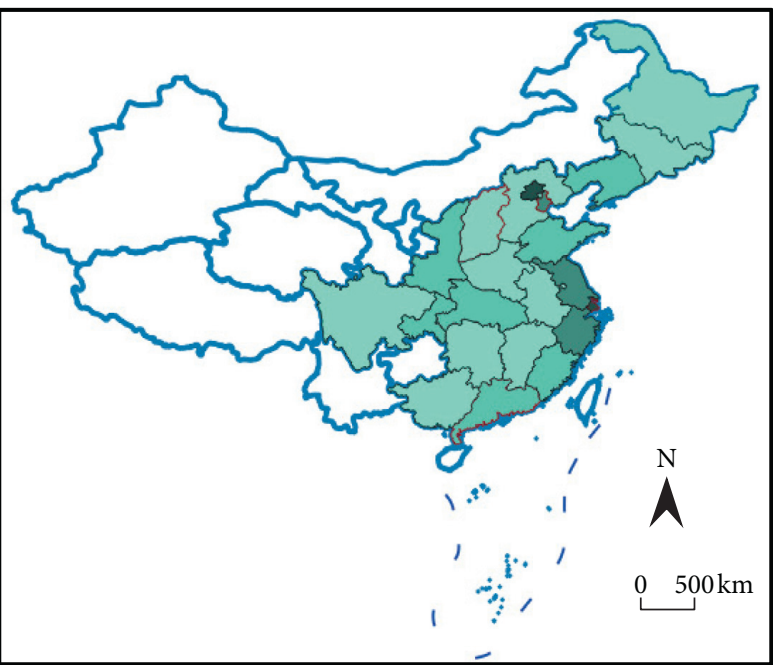

Economic-elderly services

$\square 0.2 \leq D<0.4$
$\square 0.4 \leq D<0.6$

$0.6 \leq D<0.8$

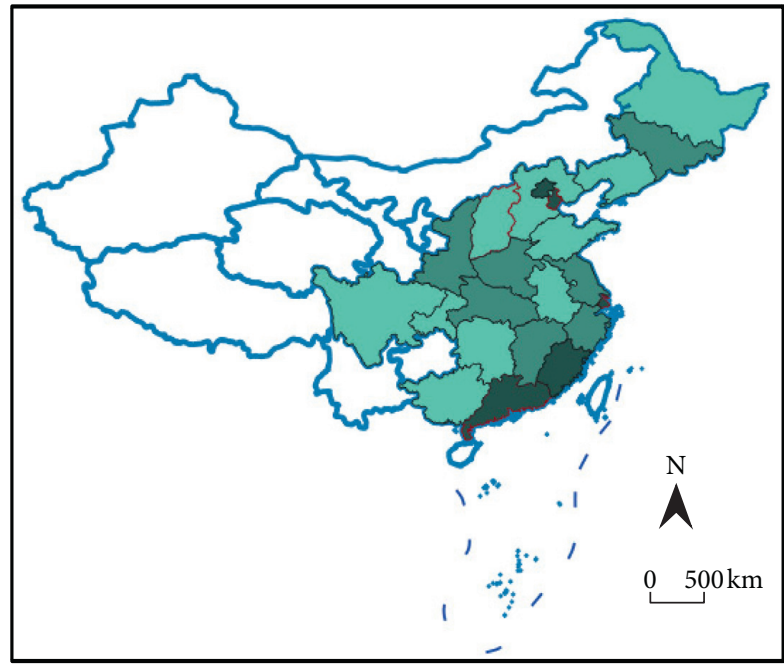

Elderly services-aging - economic

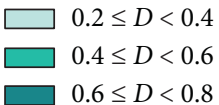

FIGURE 4: Regional characteristics of coordination degree.

coordination degree may be due to insufficient development of elderly services (e.g., SC) or poorly coordinated development (e.g., SX).

\section{Conclusions and Enlightenments}

As a public service project supported by government finance under the background of China's aging before getting rich, the development of the elderly services should not only match the degree of aging, but also coordinate with the level of local economic development. Based on the theories of matching and coordination, after calculating the index for elderly services, this paper surveys matching and coordination in 22 provinces (cities) from 2009 to 2018. The major conclusions are as follows: first, matching in elderly services-aging is improving, but more than one-fourth of the regions are mismatched, and only a small proportion has remained at a good coordination while more than $65 \%$ has remained at the basic coordination level. The degree of aging and economic level has different role in promoting the development of the elderly services, which may lead to regional differences in coordination. For example, GD and GX with a low coordination degree have a low aging level, and BJ, JS, and SH with high coordination degree have a high aging and a high economic level. With increasingly intensified aging and improving economic level in the future, 
coordination degree in elderly services-aging will be increased. Second, matching in economic level-elderly services varied according to region, with eastern provinces (cities) being better than those in the west, and more than half of provinces (cities) have remained at negative high and medium sections. Similar to the matching distribution, coordination degree shows the same regional effects since most provinces (cities) with a lower coordination are in the midwest, while the eastern region has higher coordination. This decrease in coordination from the east to the west is in line with the economic level, indicating that the economic level is still a decisive factor to the development of elderly services. Third, according to the analysis results in elderly services-aging-economic level, the matching degree is high in five provinces including GD, AH, HUB, HUN, and SC, but SX, SAX, and HLJ have positive high mismatch, indicating that these provinces have overdeveloped elderly services relative to less economic level. JS, SD, and CQ have negative high mismatch, indicating that the underdeveloped elderly services do not match the local population aging degree. Overall coordination has remained at the basic level and above, but the proportion reaching a good coordination degree and above has decreased in recent years, which may be due to the development of elderly services lagging behind the speed of aging, e.g., SD and ZJ.

Enlightenments are as follows based on the preceding conclusions: first, the coordination awareness among elderly services, aging, and economic level should be increased, and the development strategy for elderly services should be planed according to regional actual conditions rather than "quantitative" or "scaled" development goals, and overdevelopment or underdevelopment could lead to disordered and ineffective resources allocation. Second, since elderly services is a public service relying on effective economic support, and the economic level varies greatly among regions, elderly services should be attached to regional heterogeneity to formulate soft constraints and allow a lower minimum limit and greater flexibility range. For developed provinces (cities), a higher goal for the development of elderly services could be set, while the central and western regions should develop appropriate plans for elderly services to fit local conditions. Third, the high correlation between the spatial pattern of elderly services and regional economic level indicates that a good economic conditions can be a prerequisite for sustainable development of elderly services in China. The level of aging in China is far ahead of its current economic development, especially in certain areas, and it is estimated that the pressure of aging will peak around 2040 [25], so local governments should proactively respond to aging population and provide strong material support.

This paper discusses the matching and coordination in the elderly services-aging-economic level at the regional level, which provides a new perspective for evaluation of the elderly services. However, the following deficiencies have been identified: first, comprehensive and quantitative research on elderly services is still in its early stages, and currently there is no unified evaluation system. Furthermore, restricted by data availability, some indicators such as community health management of the elderly, participation in politic affairs, and other indices have not been considered. Improvement of the index system will be the focus of subsequent research. Second, considering the availability of data, this paper uses a macro perspective to analyze at the provincial level. From integrating medium and micro perspectives in evaluation, further discussion is needed in subsequent research. Third, only economic level was considered for regional differences in the development of elderly services, and other factors, such as government policy, industry policy, and improved industrial structure, will be considered in subsequent research.

\section{Data Availability}

Data are available upon request.

\section{Conflicts of Interest}

The authors declare no conflicts of interest.

\section{Acknowledgments}

This study was funded by Jiangsu Federation of Philosophy and Social Sciences and the project titled Matching and Coordination among Elderly Services, Aging, and Economic in Jiangsu (Number: 20SYB-109). Also, this work was supported by the Ministry of Science and Technology under Grant MOST 106-2410-H-182 -004 and Chang Gung Medical Foundation under Grant BMRPA79.

\section{References}

[1] National Congress, The 19th National Congress of the Chinese Government Work Report, http://www.people.com.cn/, 2017.

[2] Actively Responds to the Mid- and Long-Term Plan for Chinese Population Aging, http://www.people.com.cn/, 2019.

[3] P. X. Chen, "Fully promote the cause of aging with Chinese characteristics," Qiu Shi, vol. 2, pp. 37-39, 1994.

[4] J. W. Dang, "On the theoretical basis of dealing with China's population aging," Population Research, vol. 3, pp. 62-67, 2012.

[5] P. Du, J. J. Sun, and W. J. Zhang, "The demands of old-age care and the family and social resources for the Chinese elderly: a study based on 2014 China longitudinal aging social survey," Population Research, vol. 6, pp. 49-61, 2016.

[6] Z. G. Zheng and J. H. Lu, "The relation definition of concepts related aging services and industry under Chinese context," Scientific Research on Aging, vol. 1, pp. 57-65, 2017.

[7] S. Y. Wang, "Promoting research on aging at a newer and higher starting point," Scientific Research on Aging, vol. 1, pp. 3-14, 2018.

[8] Z. Y. Fan and S. L. Wang, "Comparative study on the development of aging services in provinces of China," Contemporary Economic Management, vol. 11, pp. 44-48, 2012.

[9] L. L. Ren, Research on the Development Indicator System of Undertakings of Aging in China, Intellectual Property Publishing House, Beijing, China, 2017.

[10] H. B. Wang and W. J. Dong, "Research on the quality of life of the elderly in Zhuhai city," Population Journal, vol. 4, pp. 60-65, 2012. 
[11] R. J. Ao, H. C. Li, and Z. Yang, "Spatial differentiation and influencing factors of health level of the elderly population: a case study of Hubei province," Progress in Geography, vol. 10, pp. 218-1228, 2017.

[12] Y. N. Liu, "Integration and innovation of pension model under the outline of collaborative development of Beijing, Tianjin and Hebei Province," Chinese Public Administration, vol. 7, pp. 132-137, 2017.

[13] X. Yuan, "A national strategy of actively responding to population aging in the new era," Population Research, vol. 5, pp. 3-8, 2018.

[14] T. G. Zeng, Y. Zhao, and X. Xu, "Research on spatial evolution pattern of hyper-aged population and its influence factors in China," Geography and Geo-Information Science, vol. 6, pp. 72-79, 2017.

[15] D. Lei, "Research on the coordination relationship between urbanization and the air environment: a case study of the Area of Wuhan,” Atmosphere, vol. 6, pp. 1539-1558, 2015.

[16] Y. Li, Y. Li, Y. Zhou, Y. Shi, and X. Zhu, "Investigation of a coupling model of coordination between urbanization and the environment," Journal of Environmental Management, vol. 98, pp. 127-133, 2012.

[17] C. Q. Song and C. X. Cheng, "Understanding geographic coupling and achieving geographic integration," Acta Geographica Sinica, vol. 1, pp. 3-133, 2020.

[18] B. Li, "Dynamic evolution and mechanism analysis of spatial dislocation of China's tourism economy," Management World, vol. 5, pp. 172-176, 2018.

[19] Y. Zhang, Y. J. Pu, and L. T. Chen, "Urbanization and Service Industry Agglomeration-A view based on system coupling interaction," China Industrial Economics, vol. 6, pp. 57-69, 2013.

[20] R. Yang, Y. S. Liu, and H. L. Long, "The study on non-agricultural transformation co-evolution characteristics of population-landindustry: case study of the Bohai Rim in China," Geographical Research, vol. 30, pp. 475-486, 2015.

[21] C. Wang and N. Tang, "Spatio-temporal characteristics and evolution of rural production living ecological space function coordination in Chongqing Municipality," Geographical Research, vol. 6, pp. 1100-1114, 2018.

[22] Y. Zhou, X. J. Huang, and G. L. Xu, "The coupling and driving forces between urban land expansion and population growth in Yangtze River Delta," Geographical Research, vol. 2, pp. 313-324, 2016.

[23] J. L. Zhu and Z. L. Li, "Coordination development of industrypopulation-space in city cluster of Yangtze River-Delta Region," China Population, Resources and Environment, vol. 2, pp. 75-81, 2015.

[24] W. N. YangZ. H. Zhang et al., "Coupling relations among population migration, technological innovation and industrial upgrading system in the New Normal Period," Urban Development Studies, vol. 10, pp. 76-85, 2019.

[25] M. Long, "Quantitative research on the coordination of population aging and economic development in China from 1980 to 2050," Population Research, vol. 3, pp. 10-19, 2009.

[26] F. H. Bao and Y. Chen, "Study on the spatial mismatch between foreign direct investment and inbound business tourism in Chinese Mainland," Journal of Zhejiang University: Science Edition, vol. 4, pp. 465-475, 2016. 